\title{
Hybrid Extensive Air Shower Detector Array at the University of Puebla to Study Cosmic Rays
}

\author{
O. Martínez ${ }^{\mathrm{a}}$, E. Moreno ${ }^{\mathrm{a}}$, G. Pérez ${ }^{\mathrm{a}}$, H. Salazar ${ }^{\mathrm{a}}$ and L. Villaseñor ${ }^{\mathrm{a}} \mathrm{b}$ \\ (a) Facultad de Fisico-Matematicas, Benemerita Universidad Autonoma de Puebla, Puebla, Pue., 72000, Mexico \\ (b) Instituto de Fisica y Matematicas, Universidad Michoacana de San Nicolas de Hidalgo, Morelia, Mich., 58040, \\ Mexico \\ Presenter: L. Villaseñor (villasen@ifm.umich.mx), mex-villasenor-L-abs1-he12-poster
}

We describe the performance of a hybrid extensive air shower (EAS) detector array built on the Campus of the University of Puebla $(19 \mathrm{oN}, 90 \mathrm{oW}, 800 \mathrm{~g} / \mathrm{cm} 2)$ to measure the energy, arrival direction and composition of primary cosmic rays with energies around the knee of the cosmic-ray energy spectrum. The array consists of 6 water Cherenkov detectors of $1.86 \mathrm{~m} 2$ cross section and 12 liquid scintillator detectors of $1 \mathrm{~m} 2$ distributed in a square grid with a detector spacing of $20 \mathrm{~m}$ over an area of $4000 \mathrm{~m} 2$. We discuss and report on measurements and reconstruction of the LDF for the electromagnetic and muonic components of extensive air showers. We also discuss the ways in which the hybrid character of the array can be used to study inclined EASs, i.e., zenithal angle $>60$ degree; and also to measure mass composition of the primary cosmic rays, i.e., by estimating the relative contents of muons with respect to the EM component.

\section{Introduction}

The hybrid extensive air shower detector array at UAP (EAS-UAP) was designed to measure the lateral distribution of the EM and muonic components of secondary particles for EAS in the energy region of $10^{14}$ $10^{16} \mathrm{eV}$. These measurements allow a determination of the arrival direction, energy and mass composition of the primary cosmic ray for energies around the "knee" of the spectrum at around $\mathrm{E}=3 \times 10^{15} \mathrm{eV}$. One of the main experimental problems in cosmic ray physics is the measurement of primary composition at energies around and higher than the knee [1], its nature remains a puzzle despite the fact that it was discovered more than 4 decades ago [2]. The main tool to study the composition of primary cosmic rays with ground detector arrays is by measuring the ratio of the muonic to the electromagnetic component of EAS; in fact, Monte Carlo simulations show that heavier primaries give rise to a bigger muon/EM ratio compared to lighter primaries of the same energy [3]. In fact, evidence for such variations has been reported recently [4].

In this paper we report on preliminary results obtained by continuous operation over one year of the first stage of the EAS-UAP array, consisting of 12 liquid scintillator detectors and 3 water Cherenkov detectors out of the 18 and 6 planned, respectively. Throughout the operation period we have made use of the hybrid scintillator-Cherenkov nature of our array to obtain complementary data to measure the arrival direction, energy and primary mass composition of cosmic rays. The special location of the EAS-UAP array $(2200 \mathrm{~m}$ above sea level and all the facilites coming from the Campus of the University of Puebla) make it a valuable apparatus for the long term study of cosmic rays and at the same time an important training center for physics students interested in getting a first class education in Mexico in the field of cosmic rays.

\section{Experimental setup}

EAS-UAP $\left(19^{\circ} \mathrm{N}, 90^{\circ} \mathrm{W}, 800 \mathrm{~g} / \mathrm{cm}^{2}\right)$ consists of 6 water Cherenkov and 18 liquid scintillator detectors distributed on a square grid with spacing of $20 \mathrm{~m}$, as shown in Figure 1. A detailed description of the detector array can be found elsewhere [5] . 


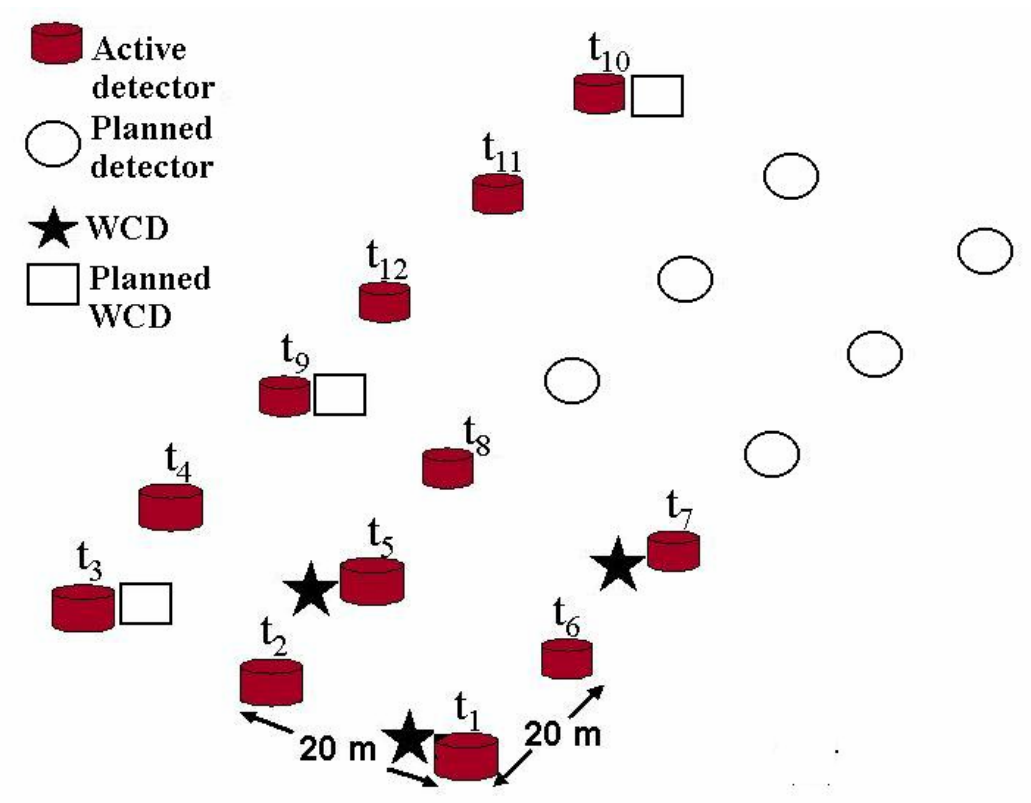

Figure 1. EAS-UAP array located on the Campus of the University of Puebla. Stars represent Cherenkov detectors filled with 22301 of water and the cylinders represent scintillation detectors filled with 130 liter of liquid scintillator.

\section{Results and discussion}

The typical dispersion rms/mean for single-particle rates monitored continouosly on all the array detectors was lower than $3 \%$ for the scintillator detectors and lower than $2 \%$ for the Cherenkov detectors. Bidimensional correlation plots, i.e., amplitud vs. risetime or charge vs risetime are also taken periodically. In fact these correlation plots can be used to separate many of the physical processes occurring inside water Cherenkov detectors (6).

We make use of the natural flux of background muons and electrons to calibrate our detectors. For the location of the EAS-UAP, muons are the dominant contribution to the flux of secondary cosmic rays for energies above $100 \mathrm{MeV}$ with a flux of about $85 \mathrm{~m}^{-2} \mathrm{~s}^{-1} \mathrm{sr}^{-1}$ and a mean energy of $4 \mathrm{GeV}$; at lower energies, up to $100 \mathrm{MeV}$, electrons, positrons and photons are also an important component. We use a DAQ system that takes calibration data simultaneously with air shower data. Figure 2 shows the spectra of charge depositions for secondary cosmic ray events triggered by a simple amplitude threshold. The plot on the left is the PMT charge for one of the 12 liquid scintillator detectors and the plot on the right for one of the four water Cherenkov detectors. Liquid scintillator detectors are more sensitive to low energy particles $(\mathrm{dE} / \mathrm{dx}$ is proportional to the inverse of the squared velocity for charged particles), while Cherenkov detectors are more sensitive to relativistic muons and electrons. While a $4 \mathrm{GeV}$ muon can fully cross the detector with a $\mathrm{dE} / \mathrm{dx}$ of around $2 \mathrm{MeV} / \mathrm{cm}$, a $10 \mathrm{MeV}$ electron has a range of about $5 \mathrm{~cm}$ in a water-like liquid. 


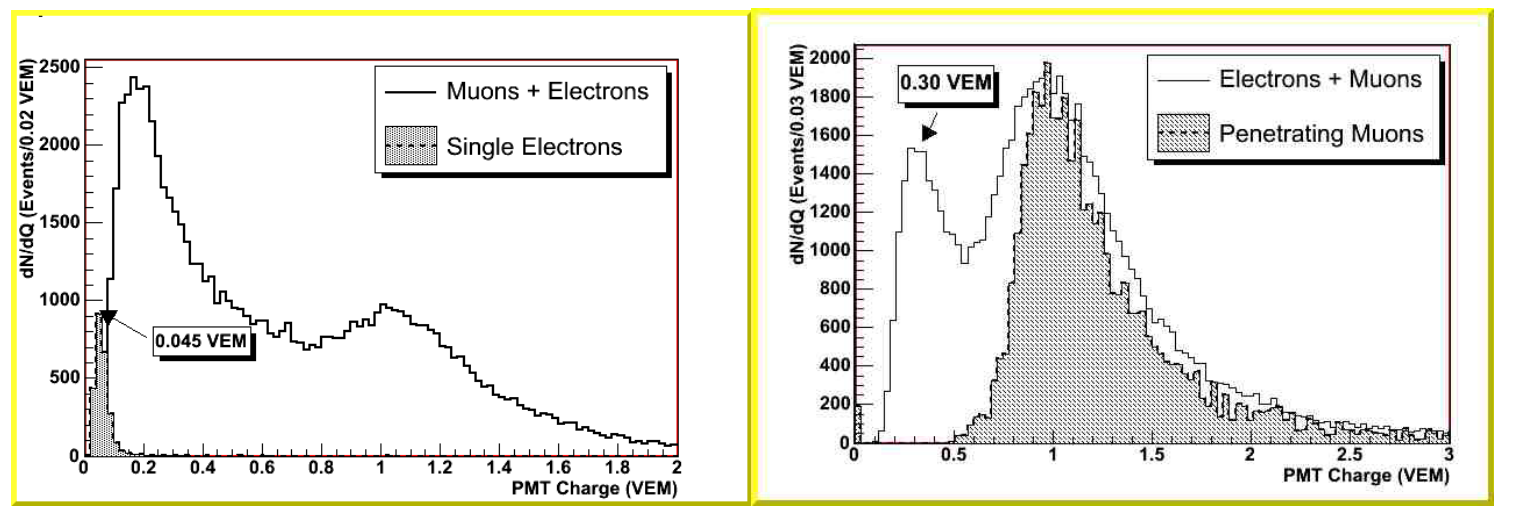

Figure 2. Spectrum of charge depositions scaled by the charge of Vertical Equivalent Muons (VEM). Left. Calibration histogram for a water Cherenkov detector filled with purified water up to a height of $1.2 \mathrm{~m}$; the shaded histogram corresponds to electrons selected by requiring $\mathrm{Q} / \mathrm{A}<0.5$ and risetime $10 \%-90 \%<0.5$. The first peak of the non-shaded histogram is dominated by corner-clipping muons, low-energy muons and electrons from muon decays; the second peak is dominated by muons crossing the whole depth of the the detector in all directions. Right: Spectrum of PMT charge deposition for one of the scintillator detectors filled with liquid scintillator up to a height of $13 \mathrm{~cm}$.

In the case of the water Cherenkov detectors the signals of crossing muons are proportional to their geometric path leghts. The second peak in the non-shaded histogram of the plot on the left of Figure 2 corresponds to the mean PMT charge deposited by muons crossing the detector in arbitrary directions, while the first peak corresponds to corner-clipping muons and also EM particles that produce smaller signals. For water Cherenkov detectors, we performed a control experiment where two scintillation hodoscopes were placed above and below the detector to allow us to determine the position of the charge peak for muons crossing the detector vertically. We call this paramenter a vertical equivalent muon (VEM). Our measurements indicate that the position of the second peak in the charge distribution of the plot on the right of Figure 2 is about 1.05 VEM. A selection of low energy electrons by requiring $\mathrm{Q} / \mathrm{A}<0.5$ and risetime $10 \%-90 \%<0.5$, gives a signal ratio muon:electron of 1:0.045, i.e., in agreement with our espectations for electrons with energies around $10 \mathrm{MeV}$ for the geometrical path lengths of $120 \mathrm{~cm}$ for vertical muons and about $5 \mathrm{~cm}$ for electrons. The plot on the right of Figure 2 corresponds to a calibration run for one of the liquid scintillation detectors. The shaded histogram gives the distribution of the PMT charge for penetrating muons triggered by a scintillator paddle placed below the scintillation detector and shielded with a $2 \mathrm{~cm}$ steel slab. The first peak of the non-shaded histogram corresponds to electrons and corner-clipping muons while the second peak is dominated by crossing muons. The ratio of the MPVs of these two peaks is about 3.3, i.e., in rough agreement with the fact that crossing muons deposit around $26 \mathrm{Mev}$ of energy in $13 \mathrm{~cm}$ of liquid while low energy electrons deposit all of their energy, i..e., around $10 \mathrm{MeV}$.

In consequence, we have a reliable way of converting the charge deposited in each detector into a number of equivalent particles, therefore we can determine the number of equivalent muons and low-energy electrons in each detector and for each EAS using the single-particle charge spectrum discussed above. The core position, lateral distribution function and total number of shower electromagnetic particles $N_{e}$ and muonic component $N_{\mu}$, are reconstructed from a fit of the the NKG expression given by $\rho(S, r)=K(S)\left(r / R_{0}\right)^{S-}$ ${ }^{2}\left(1+r / R_{0}\right)^{S-4.5}$ (7), where $S$ is the shower age, $r$ the distance of the detector to the shower core and $R_{0}$ is the Molliere radius ( $90 \mathrm{~m}$ for an altitude of $2200 \mathrm{~m}$ a.s.l.). We have measured the zenithal angular distribution (8); we found that it can be fitted by the function $A \cos ^{8} \theta \sin \theta$ using the scintillation and the Cherenkov detectors in independent ways; this distribution is in good agreement with the literature. 
Finally, the shower energy is obtained by using the relation $N_{e}\left(E_{0}\right)=99.8 E_{0}{ }^{1.15}$, where $E_{0}$ is the energy of the primary cosmic ray expressed in $\mathrm{TeV}(9)$. Analysis in progress, along with detailed simulation studies will allow us to report on mass composition.

\section{Conclusions}

A first analysis of the data collected with the EAS-UAP allows us to conclude that the detector array shows good stability. We have implemented a method of calibrating the detectors to perform the conversion of the charge deposited in each detector to vertical equivalent muons and low-energy equivalent electrons. This array has the potential to allow us to determine the lateral distribution funcion for both the electromagnetic and the muonic components with the possibility to determine not only the energy and the arrival direction, but also the mass composition of the primary cosmic ray on a event by event basis for energies around $10^{15}$ $\mathrm{eV}$. In the near future we will increase the number of water Cherenkov detectors to improve the muonic lateral distribution determination and the number of scintillator detectors to improve our aperture.

\section{Acknowledgements}

This work was partially supported by BUAP, CIC-UMSNH and CONACyT under projects G32739-E and G38706-E.

\section{References}

[1] S.P. Swordy et al., Astroparticle Physics, Volume 18 (2002) 129-150.

[2] G.V. Kulikov and G.V. Khristiansen, Sov. Phys. JETP, 41 (1959) 8.

[3] B. Alessandro, et al., Proc.27th ICRC, 1 (2001) 124-127.

[4] KASCADE Collaboration (Klages H. O. et al.), Nucl. Phys. B (Proc. Suppl.), 52 (1997) 92

[5] J. Cotzomi et al., Rev. Mex. Fis. Vol. 51 No. 1 (2005) 38-46.

[6] H. Salazar and L. Villaseñor, Separation of cosmic-ray components in a single water Cherenkov detector, in press, Nucl. Instrum. and Meths A, Proc. of the RICH2004 Conference, 2005.

[7] J. Nishimura, Handbuch der Physik XLVI/2, (1967) 1.

[8] H. Salazar, O. Martínez, E. Moreno, J. Cotzomi, L. Villaseñor, O. Saavedra, Nuclear Physics B (Proc. Suppl.) 122 (2003) 251-254.

[9] M. Aglietta et al., Phys. Lett. B, 337 (1994) 376-382. 